\title{
Time dependence of the helium flux measured by PAMELA
}

\section{B. Panico*}

INFN, Sezione di Napoli, I-80126 Naples, Italy

E-mail: beatrice.panicoana.infn.it

D. Campana, G. Osteria

INFN, Sezione di Napoli, I-80126 Naples, Italy

\section{G. C. Barbarino}

University of Naples 'Federico II', Department of Physics, I-80126 Naples, Italy

INFN, Sezione di Napoli, I-80126 Naples, Italy

O. Adriani, M. Bongi, P. Spillantini, N. Mori

University of Florence, Department of Physics and Astronomy, I-50019 Sesto

Fiorentino, Florence, Italy

INFN, Sezione di Firenze, I-50019 Sesto Fiorentino, Florence, Italy

P. Papini, E. Vannuccini

INFN, Sezione di Firenze, I-50019 Sesto Fiorentino, Florence, Italy

\section{G. A. Bazilevskaya, A. N. Kvashnin, Y. I. Stozhkov}

Lebedev Physical Institute, RU-119991, Moscow, Russia

\section{R. Bellotti, A. Monaco}

University of Bari, Department of Physics, I-70126 Bari, Italy

INFN, Sezione di Bari, I-70126 Bari, Italy

\section{A. Bruno}

University of Bari, Department of Physics, I-70126 Bari, Italy

\section{F. Cafagna}

INFN, Sezione di Bari, I-70126 Bari, Italy

M. Boezio, V. Bonvicini, E. Mocchiutti, A. Vacchi, G. Zampa, N. Zampa

INFN, Sezione di Trieste, I-34149 Trieste, Italy

\section{E. A. Bogomolov, S. Y. Krutkov, G. I. Vasilyev}

Ioffe Physical Technical Institute, RU-194021 St. Petersburg, Russia

\section{P. Carlson, M. Pearce}

KTH, Department of Physics, and the Oskar Klein Centre for Cosmoparticle Physics, AlbaNova University Centre, SE-10691 Stockholm, Sweden 


\section{Casolino}

INFN, Sezione di Roma Tor Vergata, I-00133 Rome, Italy

RIKEN, Advanced Science Institute, Wako-shi, Saitama, Japan

\section{G. Castellini}

IFAC, I-50019 Sesto Fiorentino, Florence, Italy

\section{De Santis, L. Marcelli, M. Mergé, P. Picozza, R. Sparvoli}

University of Rome Tor Vergata, Department of Physics, I-00133 Rome, Italy

INFN, Sezione di Roma Tor Vergata, I-00133 Rome, Italy

\section{Di Felice}

INFN, Sezione di Roma Tor Vergata, I-00133 Rome, Italy

Agenzia Spaziale Italiana (ASI) Science Data Center, Via del Politecnico snc I-00133 Rome, Italy

\section{R. Munini}

University of Trieste, Department of Physics, I-34147 Trieste, Italy

INFN, Sezione di Trieste, I-34149 Trieste, Italy

\section{A. M. Galper,A. V. Karelin,S. V. Koldashov,S. Koldobskiy,Y. T. Yurkin,V. Malakhov,A.} Leonov,A. G. Mayorov,V. V. Mikhailov,S. A. Voronov

National Research Nuclear University MEPhI, RU-115409 Moscow

\section{Martucci}

University of Rome Tor Vergata, Department of Physics, I-00133 Rome, Italy

INFN, Laboratori Nazionali di Frascati, Via Enrico Fermi 40, I-00044 Frascati, Italy

\section{W. Menn, M. Simon}

Universitat Siegen, Department of Physics, D-57068 Siegen, Germany

\section{Ricci}

INFN, Laboratori Nazionali di Frascati, Via Enrico Fermi 40, I-00044 Frascati, Italy

\section{S. B. Ricciarini}

INFN, Sezione di Firenze, I-50019 Sesto Fiorentino, Florence, Italy

IFAC, I-50019 Sesto Fiorentino, Florence, Italy

The last solar cycle has presented a peculiarly long quiet phase with consequent minimum modulation conditions for cosmic rays. The proton and electron spectra were measured from July 2006 to December 2009 by PAMELA experiment, providing fundamental information about the transport and modulation of cosmic rays inside the heliosphere. These studies allow to obtain a more complete description of the cosmic radiation. In this picture the time dependence of the helium spectrum become very important to constrain parameters of the actual solar modulation model. The crucial point for this analysis is the selection of a dataset of helium events which ensure high statistics with a very low contamination. In this paper the definition of the selection criteria for helium events with data taken from July 2006 to June 2014 by PAMELA experiment is reported.

The European Physical Society Conference on High Energy Physics

5-12 July, 2017

Venice 


\section{Introduction}

Although cosmic rays (CRs) have been discovered a long time ago, the mechanism of their propagation is still under study. Precise measurements of cosmic-ray spectra over a long period of time is very helpful to understand this process. When cosmic rays encounter the solar wind, they undergo a temporal variation in their intensity and in their energy as a function of the position inside the heliosphere. This process is known as the solar modulation of cosmic rays [1]. PAMELA took data from June 2006 to the beginning of 2016, covering a long part of the last solar cycle. This gives the possibility to study the influence of the solar modulation in different conditions, from the solar minimum to the maximum.

\section{The PAMELA experiment}

PAMELA (Payload for Antimatter-Matter Exploration and Light-nuclei Astrophysics) is a space-based cosmic-ray detector hosted on the Russian Resurs-DK1 satellite. It has different goals, spreading from the study of light nuclei to the antimatter, to the study of solar physics. Anyway, its layout was optimized for precision study of light particles and antiparticles in primary cosmic rays on a range of energy between tens of $\mathrm{MeV}$ and hundred of $\mathrm{GeV}$.

It was launched on June 15th of 2006 and it has taken data until the beginning of 2016. PAMELA orbit was elliptical with an inclination of about $70^{\circ}$, an altitude varying between 355 and $584 \mathrm{~km}$ and a period of about 90 minutes. In September 2010, the orbit was changed to a nearby circular one, at an altitude of about $570 \mathrm{Km}$ [4].

\section{Event Selection}

The analysis is based on data collected from June 2006 to September 2014. Selection cuts involve informations from the Tof and the tracker. Starting from the selection used in the analysis on proton and helium spectum [5], the tracking requirements have been changed to obtain a sample of events with a negligible proton contamination and an high statistics to study the variation of the low energy particle flux in different periods.

To control the charge selection with the tracker, it is important to select a pure sample of events. Thanks to the different detectors which composed PAMELA, we have a redundancy of informations. To select a sample of helium, the $\mathrm{dEdx}$ for each layer of the Tof as a function of the $1 / \beta$ is considered and a band which identifies the helium nuclei is selected on each layer.

In this analysis the fiducial area is bounded $1.5 \mathrm{~mm}$ from the magnet cavity walls and the cut to obtain an high quality track requires at least 3 hits on both $\mathrm{X}$ and $\mathrm{Y}$ view and a track lever-arm of at least 4 silicon planes in the tracker.

Finally also a cut based on the dEdx read by the silicon layers have been applied. The dEdx on the 6 layer have been summed, excluding the max value for each event. The resulting $\mathrm{dEdx}$ in the $\mathrm{X}$ and in $\mathrm{Y}$ view is reported in Fig. 1. Different particles identify different bands. As an example

\footnotetext{
* Speaker.
} 
the boundaries set to select helium are reported; in this case the contamination due to protons is less than $5 \cdot 10^{-4}$.

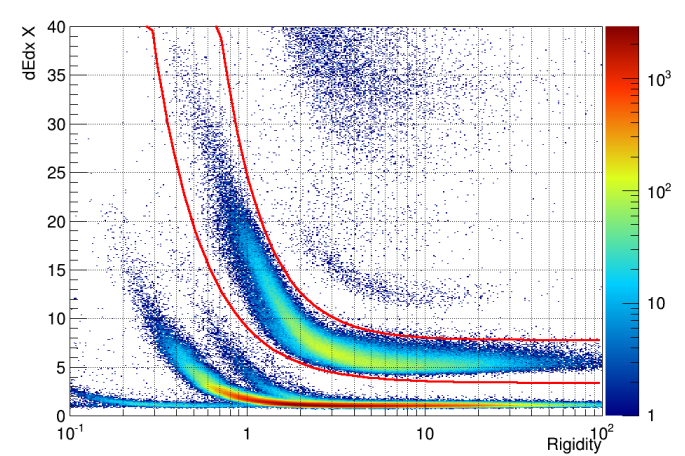

(a) $\mathrm{X}$

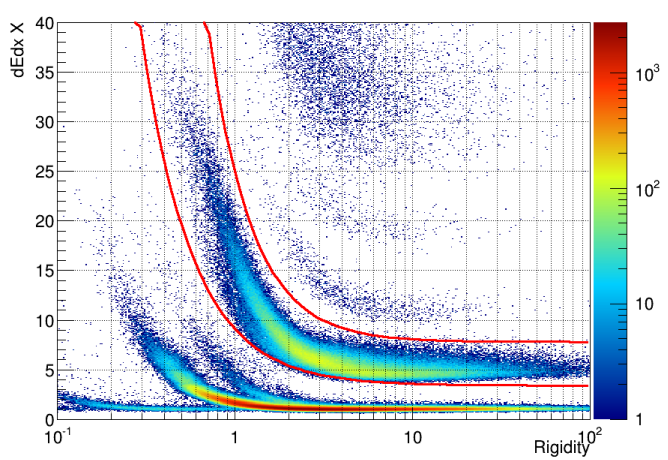

(b) $\mathrm{Y}$

Figure 1: $\mathrm{dEdx}$ on the $\mathrm{X}$ (left) and $\mathrm{Y}$ (right) view in the tracker. As example the boundaries set to select helium are reported (red lines).

\section{Conclusions}

PAMELA has studied the solar modulation of helium nuclei on a very long time from the unusual 23rd solar minimum through the following period of solar maximum activity. This gives the possibility to study the influence of the solar modulation in different conditions and also as a function of the rigidity up to a range where it is uneffective. This is fundamental to obtain informations on cosmic ray propagation parameters.

\section{Acknowledgments}

The Italian authors acknowledge the partial financial support from the Italian Space Agency (ASI) under the program 'Programma PAMELA - attività scientifica di analisi dati in fase E'.

\section{References}

[1] Potgieter, M.S., Living Rev. Sol. Phys. (2013) 10, 3, doi:10.12942/lrsp-2013-3

[2] Heber, B. \& Potgieter, M.S., Space Sci Rev (2006) 127, 117, doi:10.1007/s11214-006-9085-y

[3] Webber, W. R., McDonald, F. B., Cummings, A. C. et al., Geophys. Res. Lett. (2012) 39, 6, doi:10.1029/2012GL051171

[4] Picozza, P., Galper A., Castellini, G., Astropart. Phys. (2007) 27, 296, doi:10.1016/j.astropartphys.2006.12.002

[5] Adriani, O., Barbarino, G.C., Bazilevskaya, G.A. et al., Science (2011) 332, 6025, doi:10.1126/science. 1199172 